\title{
ENSINO DE CÔNICAS E A ARTE DAS DOBRADURAS
}

\author{
Lucas Ribeiro de Souza Tenani, Christoffer Lucas Bezão Silveira, Eugenia Brunilda Opazo Uribe
}

Universidade Federal do Mato Grosso do Sul - UFMS, PET Conexões de Saberes - Matemática, Três Lagoas, MS. Agência de fomento: Programa de Educação Tutorial. E-mail: lucastenani@hotmail.com

\section{RESUMO}

O presente trabalho faz parte de uma atividade de pesquisa desenvolvida no Grupo PET Conexões de Saberes - Matemática do CPTL/UFMS, por alunos do Curso de Licenciatura em Matemática. 0 objetivo do trabalho é explorar o ensino das Seções Cônicas de maneira a desenvolver atividades diversificadas visando a construção coletiva do conhecimento, sem deixar de lado o formalismo necessário no estudo de Matemática. As atividades desenvolvidas foram leitura e compreensão de artigos e monografias sobre o tema, os quais foram estudados através de seminários de discussão e do desenvolvimento de exercícios, demonstrações e atividades práticas utilizando o processo de dobraduras para a construção de cada uma das cônicas. As atividades práticas apresentadas podem ser desenvolvidas em sala de aula sem muita dificuldade e o estudo teórico pode fornecer subsídios para o desenvolvimento do conteúdo, bem como para o desenvolvimento de projetos.

Palavras-chave: Cônicas, Dobraduras, Geometria Analítica, Programa de Educação Tutorial.

\section{CONICAL TEACHING AND THE FOLDING ART}

\begin{abstract}
This paper is part of a research activity developed by the PET Group Knowledge Connections Mathematics of CPTL/UFMS, by Mathematics Graduation students. The goal of the paper is to explore the teaching of Conical Sections, in a manner to develop diversified activities viewing colective construction of knowledge, without leaving aside the formalism necessary in the Mathematics study. The developed activities were reading and comprehending articles and monographs about the theme, which were studied through discussion seminars and development of exercises, demonstrations and practical activities, using the folding process for the construction of each of the conics sections. The practical activities presented may be developed in class without many difficulties and the theoretical study may provide informations for the development of the content, as such for the development of projects.
\end{abstract}

Keywords: Conical, Folding, Analytical Geometry, Tutorial Education Program. 


\section{INTRODUÇÃO}

Segundo Eves (2011), a obra "Seções Cônicas", um tratado de oito volumes e aproximadamente 400 proposições, tornou famoso o matemático Apolônio, nascido em 262 a.C. no sul da Ásia Menor, já que faz um estudo exaustivo dessas curvas e supera completamente trabalhos anteriores. No Livro I de seu tratado, obtinha todas as secções cônicas da maneira hoje familiar, ou seja, a partir de um cone circular duplo, reto ou oblíquo. Os nomes elipse, parábola e hipérbole foram introduzidos por Apolônio.

As Seções Cônicas hoje são estudadas no terceiro ano de Ensino Médio e os alunos apresentam muita dificuldade no aprendizado deste tema. Assim, a atividade de pesquisa que relatamos, foi proposta no sentido de fornecer subsídios aos futuros professores para desenvolver atividades diversificadas buscando a construção coletiva do conhecimento, sem deixar de lado o formalismo necessário no estudo de Matemática. Considerando as Orientações curriculares para o Ensino Médio (Brasil, 2006) nosso trabalho partirá do princípio de que toda situação de ensino e aprendizagem deve agregar o desenvolvimento de habilidades que caracterizem o "pensar matematicamente", dando prioridade à qualidade do processo e não à quantidade de conteúdos a serem trabalhados.

O objetivo do presente trabalho é apresentar os tópicos estudados nesta atividade de pesquisa e os resultados das atividades desenvolvidas no âmbito da proposta de pesquisa do Grupo PET Conexões de Saberes - Matemática do Campus de Três Lagoas da Universidade Federal de Mato Grosso do Sul.

\section{METODOLOGIA}

O trabalho foi desenvolvido através de uma serie de pesquisas bibliográficas e de seminários apresentados para o professor orientador, realizando um estudo teórico/pratico sobre a construção das cônicas através do processo de dobraduras.

\section{RESULTADOS}

Primeiramente, apresentaremos as três seções cônicas elipse, hipérbole e parábola, através da sua definição e dos seus elementos.

\section{ELIPSE}

Definição: Dados dois pontos quaisquer do plano, $F_{1}$ e $F_{2}$, seja $2 c$ a distancia entre eles. Elipse é o conjunto dos pontos do plano cuja soma das distancias à $F_{1}$ e $F_{2}$ é a constante $2 a(2 a>2 c)$. 
Elementos da Elipse:

- $F_{1}$ e $F_{2}$ são chamados Focos,

- $C$ é o Centro da Elipse,

- $A_{1} A_{2}$ é o eixo maior,

- $B_{1} B_{2}$ é o eixo menor,

- $2 c$ é distancia focal,

- $2 a$ é medida do eixo maior,

- $2 b$ é medida do eixo menor,

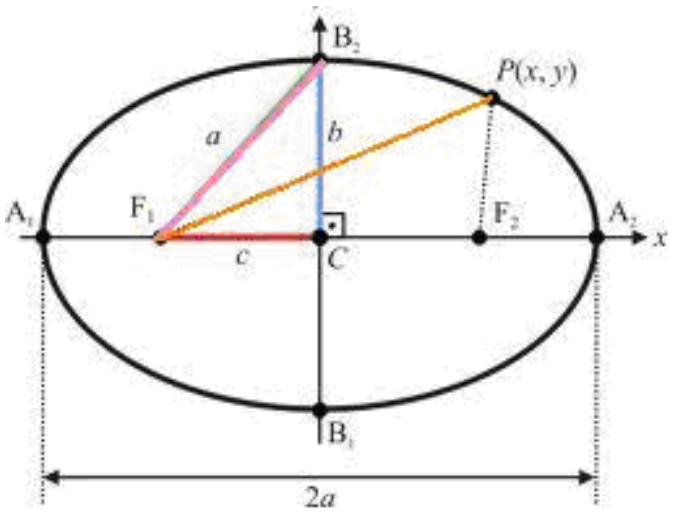

- $c / a$ é a excentricidade.

\section{Figura 1. Elipse}

Fonte: www.brasilescola.com

Os valores $a, b$ e $c$ satisfazem a relação $a^{2}=b^{2}+c^{2}$ e a equação da elipse é

$$
\frac{\left(x-x_{0}\right)^{2}}{a^{2}}+\frac{\left(y-y_{0}\right)^{2}}{b^{2}}=1,
$$

onde, $x_{0}$ e $y_{0}$ são as coordenadas do centro $C\left(x_{0}, y_{0}\right)$ da elipse.

\section{HIPÉRBOLE}

Definição: Sejam $F_{1}$ e $F_{2}$ dois pontos quaisquer do plano, seja $2 c$ a distância entre eles. Hipérbole é o conjunto dos pontos do plano cuja diferença (em módulo) das distâncias à $F_{1}$ e $F_{2}$ é a constante $2 a(0<2 a<2 c)$.

Elementos da Hipérbole:

- $F_{1}$ e $F_{2}$ são os focos da hipérbole,

- $O$ é o centro da hipérbole,

- $A_{1} A_{2}$ é o eixo real o transverso,

- $B_{1} B_{2}$ é o eixo menor ou imaginário,

- 2c é a distância focal,

- $2 a$ é a medida do eixo real ou transverso,

- $2 b$ é a medida do eixo menor ou imaginário,

- $c / a$ é a excentricidade.

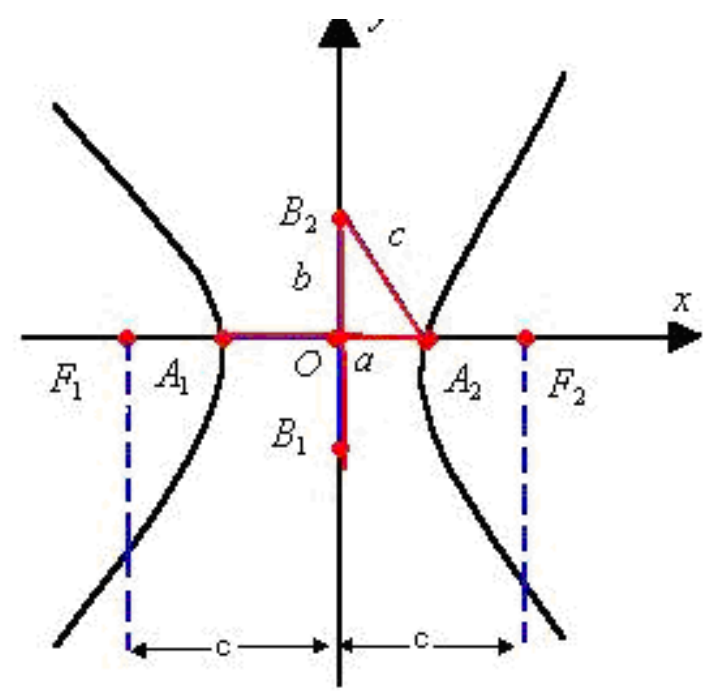

Figura 2. Hipérbole

Fonte: $\underline{w w w . b r a s i l e s c o l a . c o m}$ 
Os valores de $a, b$ e $c$ satisfazem a relação $c^{2}=a^{2}+b^{2}$ e a equação da hipérbole é

$$
\frac{\left(x-x_{0}\right)^{2}}{a^{2}}-\frac{\left(y-y_{0}\right)^{2}}{b^{2}}=1
$$

onde, $x_{0}$ e $y_{0}$ são as coordenadas do centro $C\left(x_{0}, y_{0}\right)$ da hipérbole.

\section{PARÁBOLA}

Definição: Dados um ponto $F$ uma reta $d$, do plano, com $F$ não pertencente a $d$. Parábola é o conjunto dos pontos do plano que estão a mesma distância de $\mathrm{F}$ e $d$.

Elementos da Parábola:

- F é o foco da parábola,

- $d$ é a iretriz,

- $V$ é o vértice da parábola,

- VF é o eixo de simetria,

Figura 3. Parábola

Fonte: www.brasilescola.com

A equação da parábola é dada por

$$
\left(y-y_{0}\right)^{2}=2 p\left(x-x_{0}\right)^{2},
$$

onde, $x_{0}$ e $y_{0}$ são as coordenadas do vértice $V\left(x_{0}, y_{0}\right)$ da parábola.

\section{CONSTRUCCÃO DAS CONICAS COM DOBRADURAS}

Para construção de cada uma das cônicas, devemos ter em mãos: folha vegetal tamanho A4, régua, compasso, lápis e borracha. E devemos seguir alguns passos conforme mostrado a seguir para cada uma das delas.

\section{ELIPSE}

$1^{\circ}$ Passo: Desenhe uma circunferência de raio $r$ e de centro $F_{1}$ e marque um ponto $F_{2}$ interior a mesma. Ainda, marque sobre a circunferência de raio $r, n$ pontos $P_{n}$ ( figura 4a).

20 Passo: Faça a primeira dobra de maneira que o ponto $F_{2}$ coincida com o ponto $P_{1}$ feito na circunferência. Desdobre a folha, voltando-a para a posição inicial. Temos assim o primeiro vinco, onde se deve traçar a reta $M_{1}$ (figura $4 b$ ). 
a)

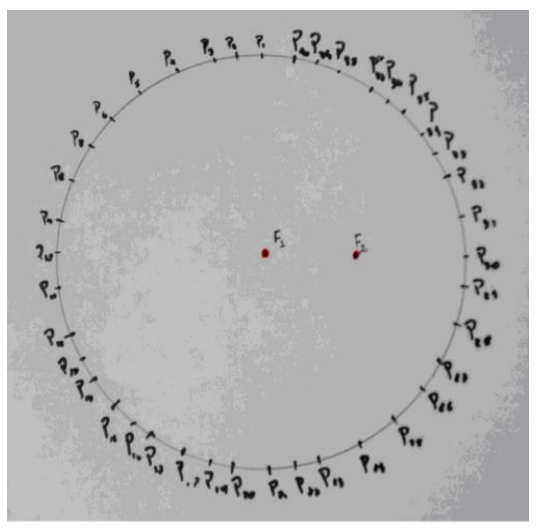

b)

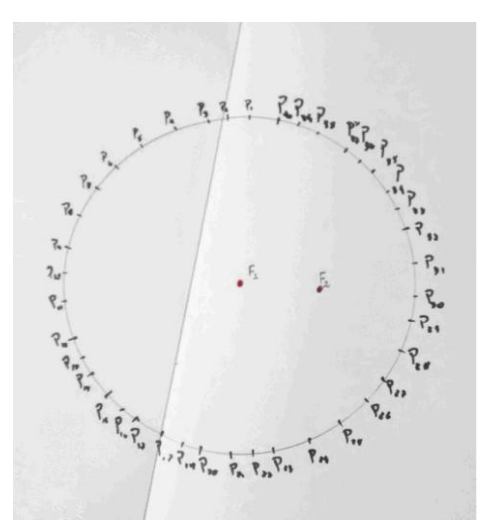

c)

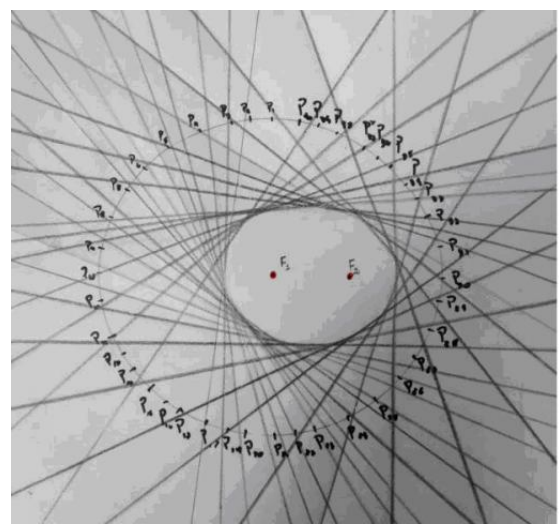

\section{Figura 4.}

Fonte: Elaborada pelos autores

3o Passo: Faça isso para todos os pontos da circunferência, assim obteremos as mediatrizes $M_{n}$ dos segmentos $F_{2} P_{n}$ e como consequência a elipse de focos $F_{1}$ e $F_{2}$ (figura $4 c$ ).

\section{HIPERBOLE}

10 Passo: Desenhe uma circunferência de raio $r$ e de centro $F_{1}$ e marque um ponto $F_{2}$ exterior a mesma. Ainda, marque sobre a circunferência de raio $r, n$ pontos $P_{n}$ (figura 5a).

20 Passo: Faça a primeira dobra de maneira que o ponto $F_{2}$ coincida com o ponto $P_{1}$ feito na circunferência. Desdobre a folha, voltando-a para a posição inicial. Temos assim o primeiro vinco, onde se deve traçar a reta $M_{1}$ (figura $5 b$ ).

a)

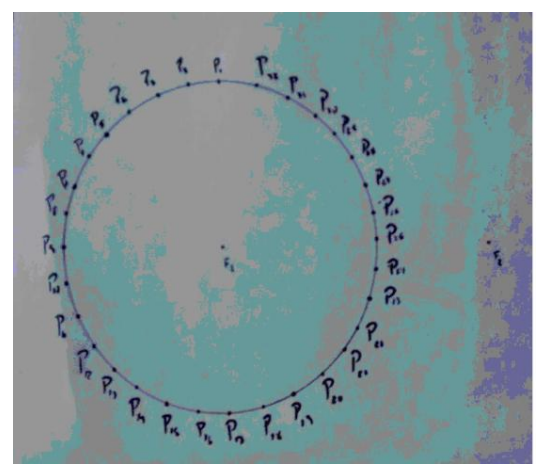

Figura 5.

Fonte: Elaborada pelos autores b)

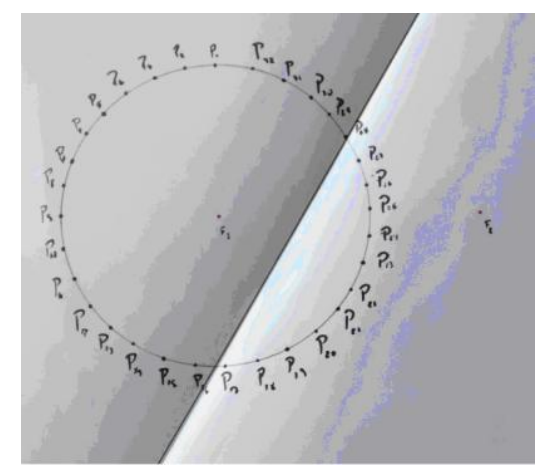

c)

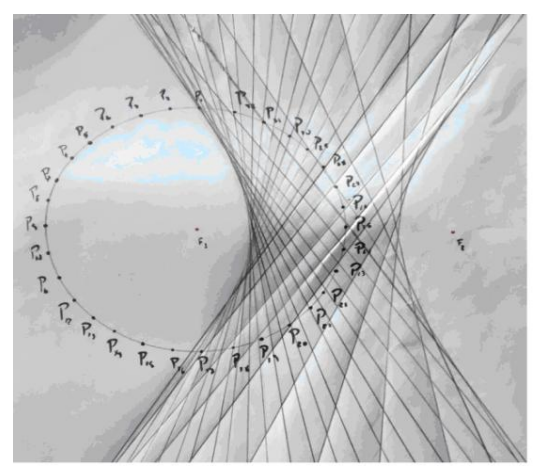


30 Passo: Faça isso para todos os pontos da circunferência, assim obteremos as mediatrizes $M_{n}$ dos segmentos $F_{2} P_{n}$ e como consequência a hipérbole de focos $F_{1}$ e $F_{2}$ (figura $5 \mathrm{c}$ ).

\section{PARABOLA}

10 Passo: Trace uma reta $d$ e marque um ponto $\mathrm{F}$ fora da mesma. Marque ainda, $n$ pontos $P_{n}$ sobre a reta $d$, (figura 6a).

20 Passo: Faça a primeira dobra de maneira que o ponto $F$ coincida com o ponto $P_{1}$. Desdobre a folha, voltando-a para posição inicial. Assim, formando o primeiro vinco, onde se deve traçar a reta $M_{1}$ (Figura 6b).

3o Passo: Faça isso para os demais pontos da reta, assim, obteremos a mediatriz $M_{n}$ dos segmentos $F P_{n}$ e como consequência a parábola de diretriz $d$ e foco $F$ (figura $6 \mathrm{c}$ ).

a)

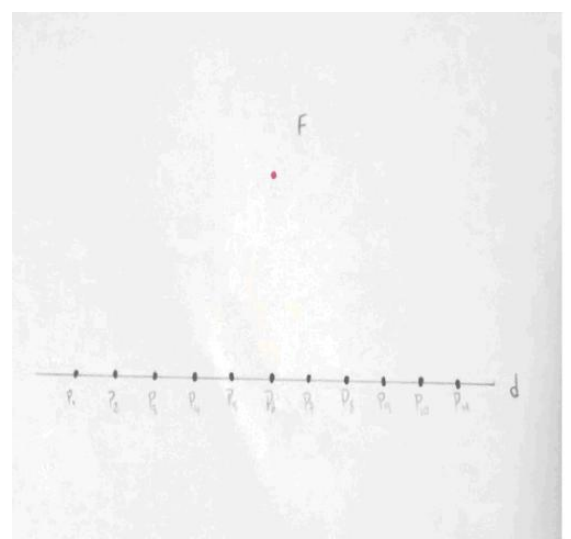

Figura 6.

Fonte: Elaborada pelos autores

\section{DISCUSSÃO}

Pode ser demonstrado que cada uma das cônicas construídas com as dobraduras satisfaz as propriedades e definições das mesmas, o que faremos a seguir para o caso da elipse.

\section{ELIPSE}

$\mathrm{Na}$ figura 7 temos uma circunferência de raio $r$ e centro $F_{1}$ e uma elipse interna a circunferência de focos $F_{1}$ e $F_{2}$. O ponto $A$ pertencente à elipse é o ponto de intersecção entre a reta $\mathrm{m}_{\mathrm{n}}$, que é a mediatriz do segmento $F_{2} P_{n}$, com o segmento $F_{1} P_{n}$. 
Queremos mostrar que $A F_{1}+A F_{2}$ é uma constante para qualquer $A$ tangente a elipse, satisfazendo assim a definição de elipse.

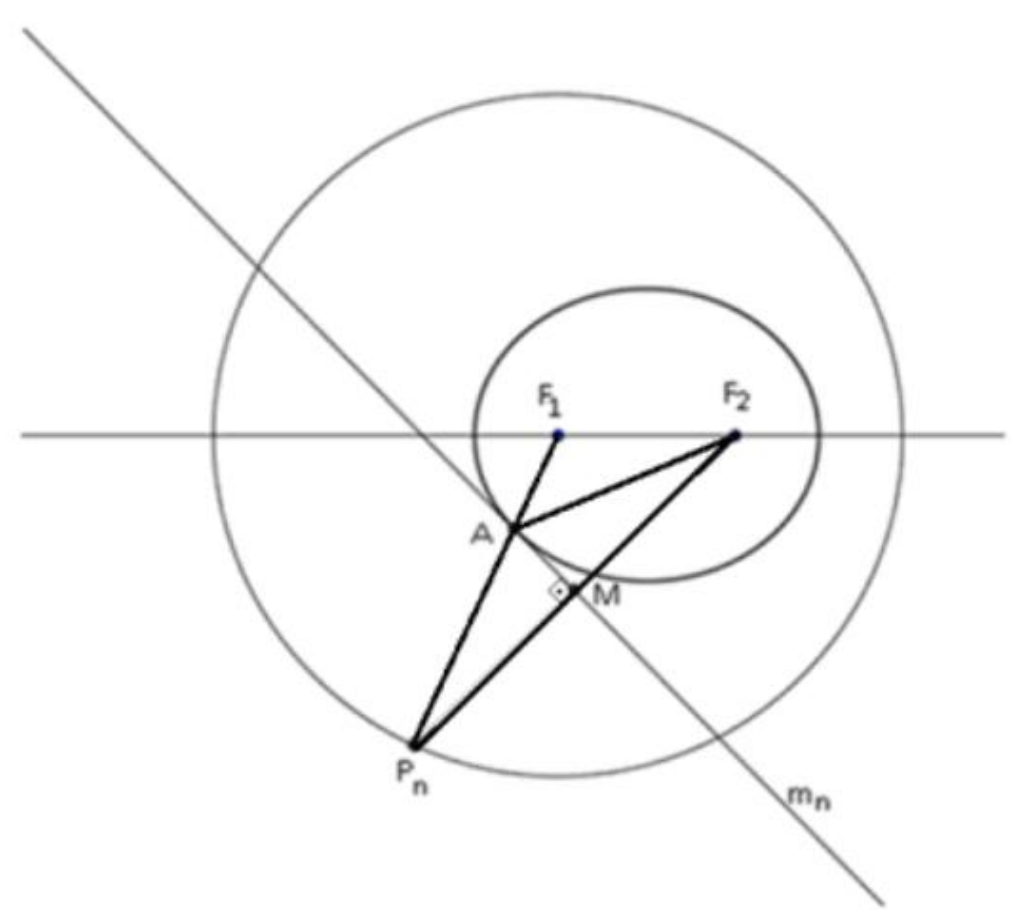

\section{Figura 7}

Fonte: Ribeiro, 2014

De fato, como dizemos antes a reta $m_{n}$ é a mediatriz do segmento $F_{2} P_{n}$, e portanto $M$ é ponto médio de $F_{2} P_{n}$ e por consequência $M P_{n}=M F_{2}$. Como o segmento $A M$ é comum aos triângulos $A M P n$ e a $A M F_{2}$, temos que $M P n=M F_{2}$ e o ângulo $P n M A$ é igual ao ângulo $A M F_{2}$, podemos concluir pelo critério LAL temos uma congruência entre esses triângulos. Assim podemos concluir que o segmento $A F_{2}$ e $A P_{n}$ são congruentes. Portanto,

$$
A F_{1}+A F_{2}=A F_{1}+A P_{n}=r \text { e que } r=2 a,
$$

(o semi eixo maior é igual ao raio da circunferência) como queríamos demonstrar.

\section{CONCLUSÃO}

Podemos concluir que os objetivos foram alcançados já que o trabalho teórico/prático proposto foi desenvolvido, com resultados muito positivos, porque foi constatado que as atividades podem ser desenvolvidas de fato em sala de aula, ajudando a diversificar as atividades para o ensino das cônicas. Além disso, os alunos participantes da atividade poderão utilizar os conteúdos desenvolvidos na sua futura vida profissional. Como continuidade deste projeto está 
sendo trabalhado o uso de artefatos para a construção de cônicas, isto é, pesquisa de materiais concretos para complementar o ensino de cônicas em Laboratório de Ensino.

\section{REFERENCIAS}

1. BRASIL. Ciências da natureza, matemática e suas tecnologias / Secretaria de Educação Básica. Brasília: Ministério da Educação, Secretaria de Educação Básica, 2006. 135 p. (Orientações curriculares para o ensino médio; volume 2).

2. EVES, H. Introdução à História da Matemática. Tradução Hygino H. Domingues. 5a ed. Campinas, SP: Editora da Unicamp, 2011.

3. HARTUNG, G.E, Construção das Cônicas com Dobraduras. Portal do Professor.

Disponível em: http://portaldoprofessor.mec.gov.br/fichaTecnicaAula.html?aula=27219

4. RIBEIRO, F.O. O Uso de Dobraduras no Processo de Ensino das Cônicas no Ensino Básico. Monografia (PROFMAT). Departamento de Ciências Exatas. Universidade Estadual de Santa Cruz.. 2014.

5. Brasil Escola. Disponível em http://www.brasilescola.com/matematica 